\title{
A meta-analysis of the association between Behçet's disease and MICA-A6
}

\author{
FEIRAN WEI ${ }^{1}$, YU ZHANG ${ }^{1}$ and $\mathrm{WEI} \mathrm{LI}^{2}$ \\ ${ }^{1}$ Clinical Medical College, Yangzhou University, Yangzhou, Jiangsu 100044; ${ }^{2}$ Department of Infectious Disease Prevention \\ and School Health, Nanjing Municipal Center for Disease Control and Prevention, Nanjing, Jiangsu 210009, P.R. China
}

Received September 21, 2015; Accepted February 1, 2016

DOI: 10.3892/br.2016.644

\begin{abstract}
Behçet's disease (BD) is a multi-system inflammatory condition with unknown cause, characterized by a triple-symptom complex of recurrent oral aphthous ulcers, genital ulcers, and uveitis and skin lesions. It predominantly affects people living around the Mediterranean basin and in Japan. The effects of the major histocompatibility complex class I chain related gene A (MICA) A6 allele on susceptibility to Behçet's disease (BD) have been reported previously, however, their results have been unreliable. The present study aimed to determine whether an association between the MICA-A6 allele and BD susceptibility exists. A total of 12 case-control studies, examining the association between MICA-A6 and BD and involving 752 cases and 1,175 controls were included in the present meta-analysis. Adjusted odds ratios (ORs) and 95\% confidence intervals (CIs) were calculated. The results of meta-analysis revealed that the frequency of the MICA-A6 allele in the case group was significantly higher than those in the control groups $(\mathrm{P}<0.001, \mathrm{OR}=2.43$, 95\% CI: 1.99-2.97). Sub-group analysis by ethnicity indicated that the association between of MICA-A6 gene to BD remained in Asian populations (5 cases and 731 controls) and Caucasian populations (242 cases and 444 controls) with $\mathrm{OR}=2.65,95 \% \mathrm{CI}: 2.07-3.38$ and $\mathrm{OR}=2.23$, 95\% CI: $1.37-3.62$, respectively. These findings demonstrate that MICA-A6 gene is associated with susceptibility to BD. The MICA-A6 gene may serve as an early diagnostic marker for BD in the future.
\end{abstract}

\section{Introduction}

Behçet's disease (BD) was first reported by Behçet in 1937. $\mathrm{BD}$ is a multi-system inflammatory disease with unknown cause, characterized by a triple-symptom complex of recurrent oral aphthous ulcers, genital ulcers, and uveitis and skin lesions (1). BD predominantly affects people living around the Mediterranean basin and in Japan (2). The etiology and

Correspondence to: Mr. Yu Zhang, Clinical Medical College, Yangzhou University, Yangzhou, Jiangsu 100044, P.R. China E-mail: yzzy10182001@aliyun.com

Key words: BD, MICA-A6, meta-analysis pathogenesis of BD is complex, as it is a polygenic disease with multiple genetic factors linked to the pathogenesis of the disease. A number of previous studies have indicated that BD is associated with environmental risk factors such as infectious agents, which are likely involved in the susceptibility to the disease (3). The HLA-B51 allele polymorphism has been associated with the pathogenesis of BD (4). Furthermore, major histocompatibility complex (MHC) class I chain related $\mathrm{A}$ (MICA) is a functional gene located between the HLA-B and tumor necrosis factor genes on the short arm of human chromosome (5). MICA is associated with autoimmune diseases, such as Ankylosing spondylitis, and is predominantly expressed in epithelial cells, keratinocytes, endothelial cells, and monocytes (6).

The MICA gene is composed of six exons, and the polymorphism located in exon 5 has at least five alleles (A4, A5, A5.1, A6 and A9) presenting 4, 5, 6 and 9 triplet repeats of (GCT/AGC) in the transmembrane (TM) region (7). The incidence of Behçet's disease is known to have a strong association with the presence of the MICA-A6 allele in Japanese patients (7). Similar results demonstrating this association were reviewed by Park et al (8). However, another previous study has shown the connection between the MICA-A6 allele and the incidence of BD is unclear (9). The reasons for these contradictory results may be caused by the sample size, which was relatively small. In order to better understand the association between the MICA-A6 gene and the incidence of BD, the present study aimed to conduct a meta-analysis. This was achieved by combining all published and unpublished work, using epidemic principles and methods to evaluate the literature and to draw conclusions about the association between the MICA-A6 gene and the incidence of BD.

\section{Materials and methods}

Search strategies. The current systemic literature review was performed by searching for literature and articles published before February 2015 in the PubMed, EMBASE and Web of Science databases. The most relevant articles were identified using medical subject headings and keywords, and search terms including MICA, polymorphism and Behçet's disease. Additional related publications were identified by a manual search of the bibliographies of the included studies to ensure that all relevant studies and recent reviews were included. 
Table I. Main characteristics of the studies included in the meta-analysis.

\begin{tabular}{|c|c|c|c|c|c|c|c|c|c|}
\hline \multirow[b]{2}{*}{ Author, year } & \multirow[b]{2}{*}{ Country } & \multirow[b]{2}{*}{ Ethnicity } & \multirow[b]{2}{*}{ Case/Control } & \multicolumn{2}{|c|}{ Gender (M/F) } & \multicolumn{2}{|c|}{ MICA-TM A6 } & \multirow[b]{2}{*}{ NOS } & \multirow[b]{2}{*}{ Refs. } \\
\hline & & & & Case & Control & Case & Control & & \\
\hline Nishiyama et al, 2006 & Japan & Asian & $23 / 23$ & $18 / 5$ & $16 / 7$ & 13 & 11 & 8 & (9) \\
\hline Mizuki et al, 2002a & Iran & Asian & $84 / 87$ & & & 64 & 53 & 8 & $(17)$ \\
\hline Park et al, 2002 & Korea & Asian & $108 / 204$ & & & 69 & 92 & 7 & $(8)$ \\
\hline Salvarani et al, 2001 & Italy & Caucasian & $69 / 130$ & $41 / 28$ & & 42 & 66 & 7 & (3) \\
\hline Gonzalez et al, 1999 & Spain & Caucasian & $58 / 194$ & & & 41 & 119 & 6 & $(12)$ \\
\hline Yabuki et al, 1999 & Greece & Caucasian & $38 / 40$ & $27 / 11$ & & 33 & 20 & 8 & (14) \\
\hline Mizuki et al, 1997 & Japan & Asian & $77 / 103$ & & & 57 & 47 & 8 & (7) \\
\hline Muzuki et al, 2001 & Jordan & Asian & $49 / 50$ & $35 / 14$ & & 43 & 31 & 7 & (16) \\
\hline Muzuki et al, 2000 & Japan & Asian & $95 / 132$ & & & 67 & 62 & 8 & $(15)$ \\
\hline Muzuki et al, 2000 & Greece & Caucasian & $55 / 52$ & & & 44 & 30 & 8 & (15) \\
\hline Muzuki et al, 2000 & Italy & Caucasian & $22 / 28$ & & & 17 & 16 & 7 & (15) \\
\hline Ota et al, 1999 & Japan & Asian & $74 / 132$ & & & 56 & 62 & 7 & (13) \\
\hline
\end{tabular}

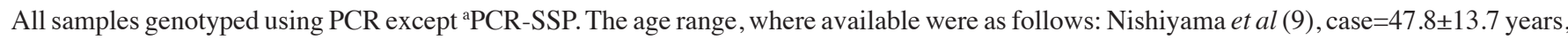
control $=40.2 \pm 13.7$ years; Park et al $(8)$, case $=41.2$ years (range, 21-62). M, male; F, female; MICA-TM A6, major histocompatibility complex class I chain related A allele 6; NOS, Newcastle-Ottawa scale.

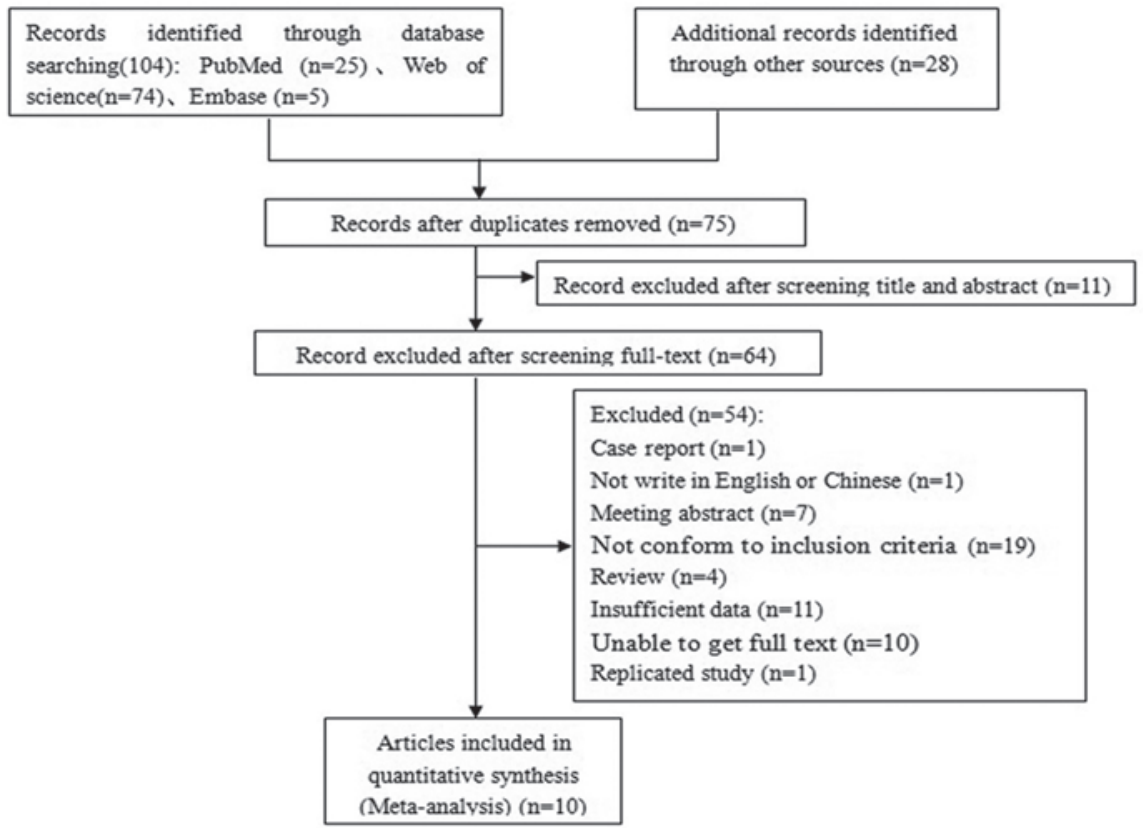

Figure 1. Flow diagram of study selection.

Inclusion and exclusion criteria. For inclusion in the present meta-analysis, the studies had to meet the following inclusion criteria: i) Study design (case-control or cohort studies; ii) subjects were diagnosed with DB; iii) studies confirmed the association between MICA gene polymorphism and the pathogenesis of Behçet's disease.

Exclusion criteria were as follows: i) Key words/search term/reviews, and abstract scanning criteria; ii) unrelated to each of BD and MICA gene; iii) research does not involve human subjects; iv) and duplication articles, unable to obtain complete full information or statistical data.
Two authors independently screened all titles and abstracts, and then applied the inclusion and exclusion criteria to the articles and evaluated their quality. Any disagreement between the authors was resolved through discussion. This process established an information extraction table, which includes the first author's name, year of publication, the study population, ethnicity, the age and gender of participants, the number of cases and controls and the method for determination of genotype.

Statistical analysis. All analyses were conducted using STATA version 12.0 software (StataCorp, College Station, TX, 


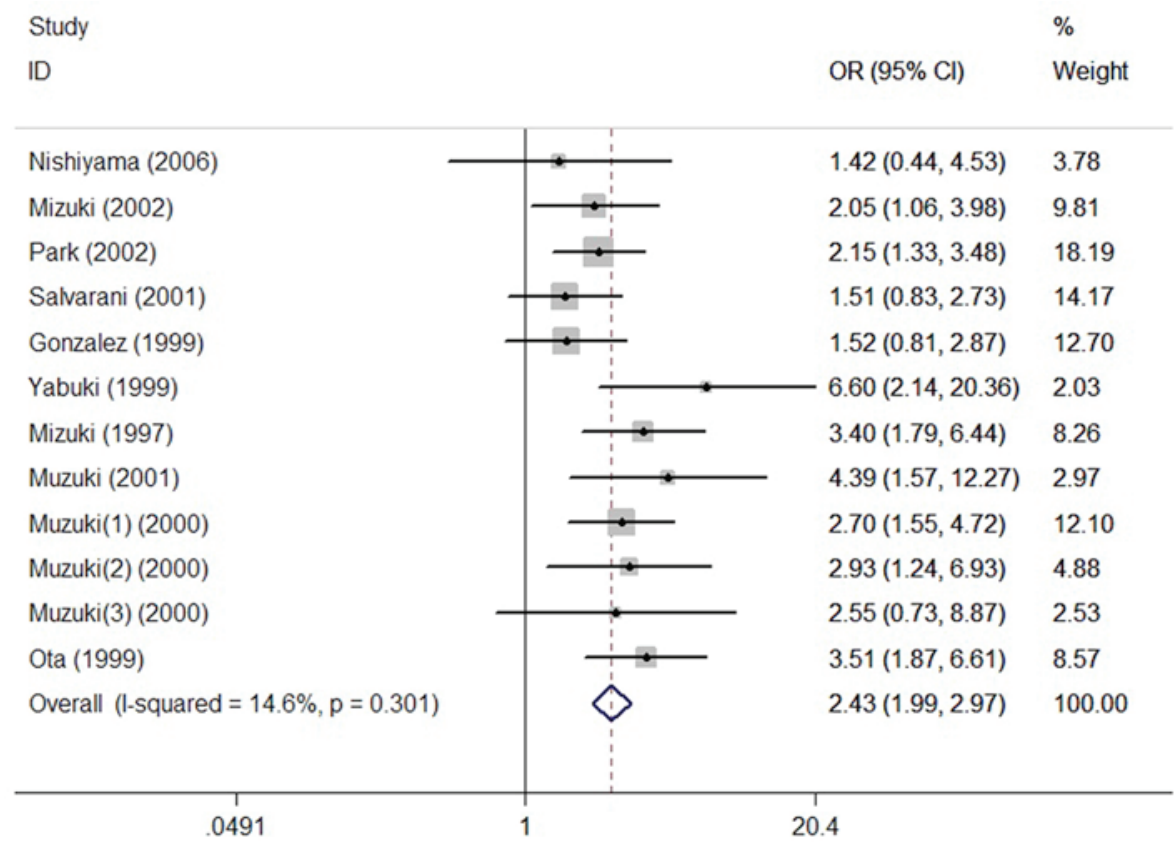

Figure 2. Association between MICA-A6 gene and Behçet's disease. OR, odds ratio; CI, confidence interval.

USA). The statistical significance of odds ratio (OR) with the corresponding $95 \%$ confidence intervals (CIs) was determined by $\mathrm{Z}$-test, and $\mathrm{P}<0.05$ was considered to indicate a statistically significant difference. Since potential causes of heterogeneity among studies include genetic differences between races, stratification analyses were conducted on ethnicity (Asian and Caucasian). The stability of the summary risk estimate was assessed using a sensitivity analysis in which each study was individually removed, and the OR was recomputed. Funnel plot, Egger's test and Begg's test were used to assess the publication bias. We estimated heterogeneity between studies using Cochran's Q and the $\mathrm{I}^{2}$ statistic $(10,11)$. We selected fixed-effects model when $\mathrm{I}^{2}<50 \%$ and used random-effects size while $\mathrm{I}^{2} \geq 50 \%$. The pooled OR was calculated by fixed effects model across studies according to the heterogeneity score.

\section{Results}

Characteristics of studies included in the meta-analysis. A total of 104 English-language articles were filtered, and in the end, 12 case-control studies in 10 articles were included in the meta-analysis (3,7-9,12-17). A flowchart of the study selection process and its results is presented in Fig. 1. A total of 10 studies were included in the article containing 752 cases and 1,175 controls. One of these articles included studies from three countries (15). Seven of the twelve studies contained patients of Asian ethnicity, and the remaining five consisted of Caucasian subjects. Data on the MICA-A6 allele was integral to these studies, and basic characteristics and quality evaluation are presented in Table I.

Meta-analysis results. In total, 12 studies reporting the relationship between MICA A6 and BD were included in the meta-analysis, with a total of 546 cases with the A6 allele in the case group and 609 patients in the control group. Cases with the allele A6 are observed as an exposure factor and those without A6 as an non-exposure factor. The result shows that there is an obvious difference between the case group and control group in the distribution of alleles A6 $\left(\chi^{2}=82.43\right.$, $\mathrm{P}<0.001)$. The fixed-effects model shows the frequency of MICA-A6 gene in case group was higher than that in the control group [OR=2.43 (1.99-2.97)] (Fig. 2).

MICA-A6 gene subgroup analysis was stratified by ethnicity. The seven studies among Asian populations included 510 cases and 731 controls. Subgroup analysis was used to calculate the odds ratio of A6 allele carriers vs. non-carriers $(\mathrm{OR}=2.65$, 95\% CI: 2.07-3.38). European populations were obtained from a total of five studies, including 242 cases, 444 controls. Subgroup analysis was used to calculate the OR of A6 allele carriers vs. non-carriers (OR=2.23, 95\% CI: 1.37-3.62) (Fig. 3).

Sensitivity analysis and publication bias. Using fixed effects and random effects models for a data-merge, the merging of two models OR and 95\%CI is comparable. The results of meta-analysis indicated the best stabilities. The analysis included 12 studies, and quantitative detection of the Egger's Funnel plot indicated no relevant publication bias. The Z Begg test was $<1.96$, and $\mathrm{P}>0.05$. The Egger's test results demonstrated $\mathrm{t}=1.09$ and $\mathrm{P}=0.303$, consistent with the funnel chart results, showing no significant publication bias (Fig. 4).

\section{Discussion}

BD is a multi-system inflammatory disease of unknown cause. It is characterized by recurrent oral aphthous ulcers, genital ulcers, uveitis, and skin lesions, and multiple genetic factors in combination with undefined environmental risk factors such as infectious agents, are important determinants of susceptibility to the disease. Studies have shown that there is a high correlation between HLA-B51 and Behcet's disease. The frequency of HLA-B51 allele in BD patients 


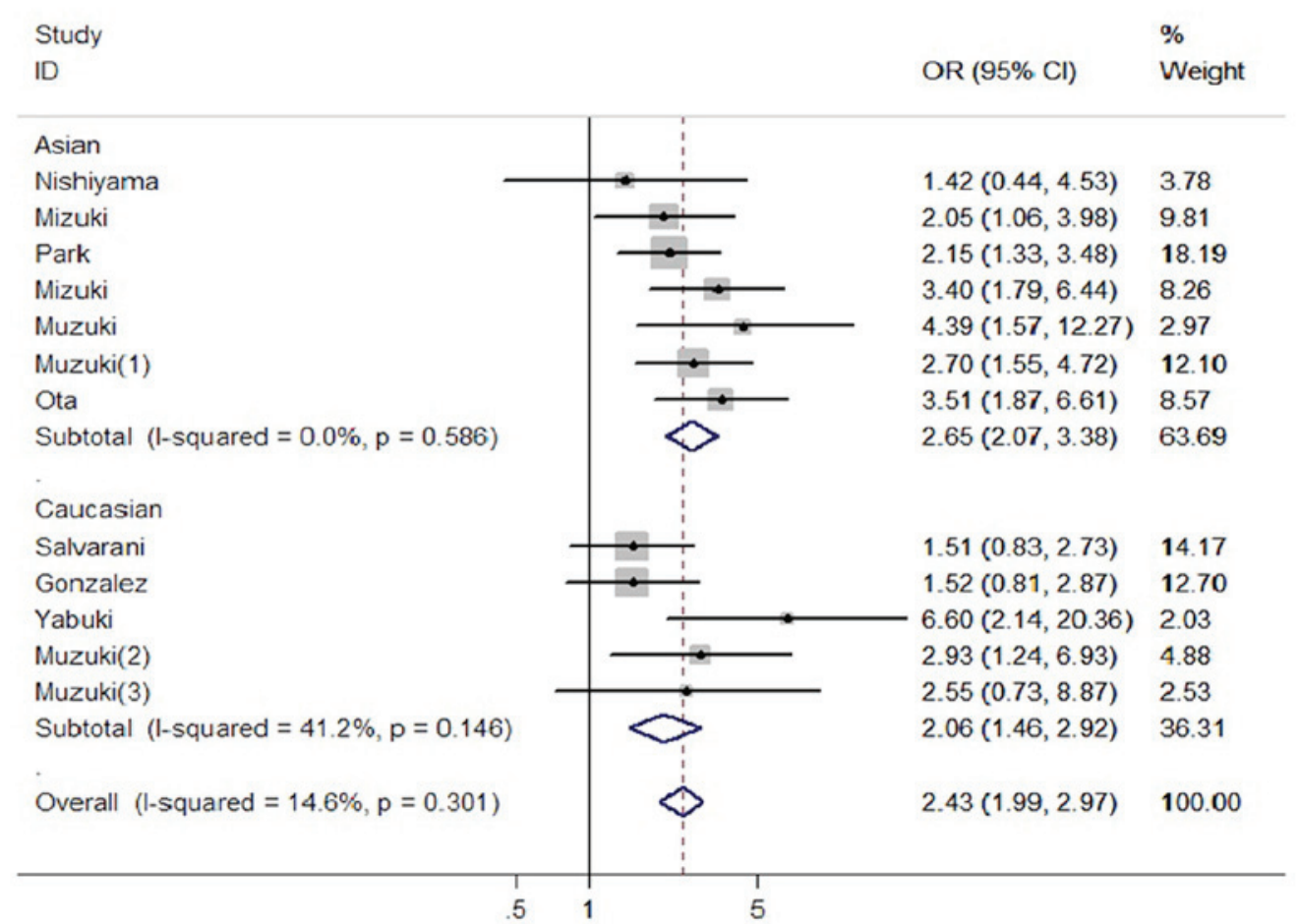

Figure 3. Association between the MICA-A6 gene and Behçet's disease by ethnicity. OR, odds ratio; CI, confidence interval.

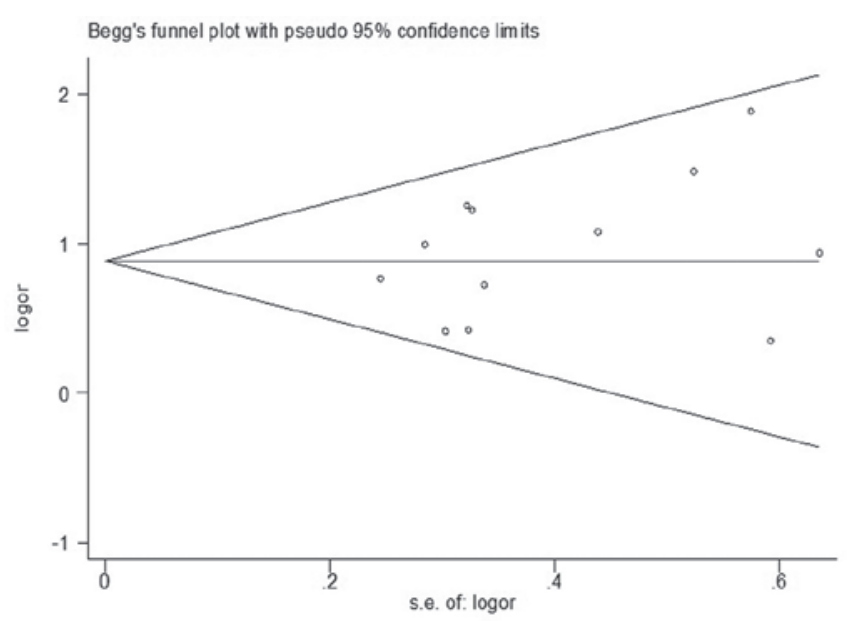

Figure 4. Begg's funnel plot for publication bias analysis of the MICA-A6 gene.

was significant higher than that of the control group (18). The MICA gene is considered as a strong candidate gene in controlling susceptibility to $\mathrm{BD}$, based on its chromosomal localization, its predicted immunologic function as a ligand of Vd1 gd T cells, its restricted and heat shock-induced expression in epithelial cells, and a strong association of this particular MICA-TM allele, MICA-A6, with BD (14). Mizuki et al (7) identified 6 microsatellite alleles repeat units composed of GCT/AGC (MICA-A6) with a strong association to BD. However Nishiyama et al (9) did not identify an association between MIC-A6 and BD. The use of different size and sample size, environmental factors and genetic variation of races may explain these findings. The present study used published case-control studies, and increased power to clarify whether the MIC-A6 allele was associated with susceptibility to $\mathrm{BD}$. The present meta-analysis demonstrates a significant association between the BD and the MICA-A6 allele, individual carriers of this allele had a 2.43 -fold higher risk of having BD than those individuals not carrying the allele. Stratified analysis shows this association remained significant in both the Caucasian and Asian study populations. It has been reported previously that MICA-A6 may tend to activate $\mathrm{V}_{\delta} 1 \gamma \delta \mathrm{T}$ cells more effectively via specific interaction with $\gamma \delta \mathrm{T}$ cells, because of either the presence of specific amino acids in the a1/a2 domains linked to MICA-A6 or that of a particular $\mathrm{V}_{\delta} 1 \gamma \delta \mathrm{T}$ cells repertoire tcan recognize the MICA molecule with MICA-A6 in an efficient way $(14,19)$. A sensitivity analysis of the present study's results following the removal of the largest studies displayed an association between the MICA-A6 allele and susceptibility to BD, giving further credibility to the findings. No significant publication bias was detected.

The present study used meta analysis to eliminate deficiencies such as the small sample size or the regional differences, however, some limitations still remain. For example the the OR value came from the data without additional checking. If we had access to the original information, more accurate results may have been obtained. In addition, there is a significant linkage disequilibrium between MICA-A6 alleles and HLA-B. In the present study, the effect on the pathogenesis of $\mathrm{BD}$ was ignored. Moreover, our research only searched several representative databases, and only used English as the retrieval language, which may influence our results.

In conclusion, the present meta-analysis demonstrates an association between the MICA-A6 allele and genetic predisposition to Behcet's disease, with individuals carrying this allele having an increased risk of BD. Therefore, the MICA-A6 allele 
may serve as an early diagnostic marker for clinical diagnosis. Future studies with larger sample size are required to confirm the conclusions presented in the present study.

\section{Acknowledgements}

The current study was supported by the National Natural Science Foundation of China (grant no. 81273143).

\section{References}

1. McGonagle D and McDermott MF: A proposed classification of the immunological diseases. PLoS Med 3: e297, 2006.

2. Günaydin I, Ustündağ C, Kaner G, Pazarli H, Yurdakul S, Hamuryudan V, Yazici Y and Yazici H: The prevalence of Sjögren's syndrome in Behçet's syndrome. J Rheumatol 21: $1662-1664,1994$

3. Salvarani C, Boiardi L, Mantovani V, Olivieri I, Ciancio G, Cantini F, Salvi F, Malatesta R, Molinotti C, Govoni M, et al: Association of MICA alleles and HLA-B51 in Italian patients with Behçet's disease. J Rheumatol 28: 1867-1870, 2001.

4. de Menthon M, Lavalley MP, Maldini C, Guillevin L and Mahr A: HLA-B51/B5 and the risk of Behçet's disease: A systematic review and meta-analysis of case-control genetic association studies. Arthritis Rheum 61: 1287-1296, 2009.

5. Bahram S, Bresnahan M, Geraghty DE and Spies T: A second lineage of mammalian major histocompatibility complex class I genes. Proc Natl Acad Sci USA 91: 6259-6263, 1994.

6. Zwirner NW, Fernández-Viña MA and Stastny P: MICA, a new polymorphic HLA-related antigen, is expressed mainly by keratinocytes, endothelial cells and monocytes. Immunogenetics 47: 139-148, 1998.

7. Mizuki N, Ota M, Kimura M, Ohno S, Ando H, Katsuyama Y, Yamazaki M, Watanabe K, Goto K, Nakamura S, et al: Triplet repeat polymorphism in the transmembrane region of the MICA gene: A strong association of six GCT repetitions with Behçet disease. Proc Natl Acad Sci USA 94: 1298-1303, 1997.

8. Park SH, Park KS, Seo YI, Min DJ, Kim WU, Kim TG, Cho CS, Mok JW,Park KS and Kim HY: Association of MICA polymorphism with HLA-B51 and disease severity in Korean patients with Behcet's disease. J Korean Med Sci 17: 366-370, 2002.
9. Nishiyama M, Takahashi M, Manaka K, Suzuki S, Saito M and Nakae K: Microsatellite polymorphisms of the MICA gene among Japanese patients with Behçet's disease. Can J Ophthalmol 41: 210-215, 2006.

10. Jackson D, White IR and Riley RD: Quantifying the impact of between-study heterogeneity in multivariate meta-analyses. Stat Med 31: 3805-3820, 2012.

11. Higgins JP, Thompson SG, Deeks JJ and Altman DG: Measuring inconsistency in meta-analyses. BMJ 327: 557-560, 2003.

12. González-Escribano MF, Rodríguez MR, Aguilar F, Alvarez A, Sanchez-Roman J and Núñez-Roldán A: Lack of association of MICA transmembrane region polymorphism and Behçet's disease in Spain. Tissue Antigens 54: 278-281, 1999.

13. Ota M, Mizuki N, Katsuyama Y, Tamiya G, Shiina T, Oka A, Ando H, Kimura M, Goto K, Ohno S and Inoko H: The critical region for Behçet disease in the human major histocompatibility complex is reduced to a 46-kb segment centromeric of HLA-B, by association analysis using refined microsatellite mapping. Am J Hum Genet 64: 1406-1410, 1999.

14. Yabuki K, Mizuki N, Ota M, Katsuyama Y, Palimeris G, Stavropoulos C, Koumantaki Y, Spyropoulou M, Giziaki E, Kaklamani V, et al: Association of MICA gene and HLA-B*5101 with Behçet's disease in Greece. Invest Ophthalmol Vis Sci 40: 1921-1926, 1999.

15. MizukiN, Ota M, Yabuki K, Katsuyama Y, Ando H,Palimeris GD, Kaklamani E, Accorinti M, Pivetti-Pezzi P, Ohno S and Inoko H: Localization of the pathogenic gene of Behçet's disease by microsatellite analysis of three different populations. Invest Ophthalmol Vis Sci 41: 3702-3708, 2000.

16. Mizuki N, Yabuki K, Ota M, Verity D, Katsuyama Y, Ando H, Onari K, Goto K, Imagawa Y, Mandanat W, et al: Microsatellite mapping of a susceptible locus within the HLA region for Behçet's disease using Jordanian patients. Hum Immunol 62: 186-190, 2001.

17. Mizuki N, Yabuki K, Ota M, Katsuyama Y, Ando H, Nomura E, Funakoshi K, Davatchi F, Chams H, Nikbin B, et al: Analysis of microsatellite polymorphism around the HLA-B locus in Iranian patients with Behçet's disease. Tissue Antigens 60: 396-399, 2002.

18. Mizuki N, Ohno S, Ando H, Chen L, Palimeris GD, et al: A strong association between HLA-B*5101 and Behçet's disease in Greek patients. Tissue Antigens 50: 57-60, 1997.

19. Yamashita N, Kaneoka H, Kaneko S, Takeno M, Oneda K, Koizumi H, Kogure M, Inaba G and Sakane T: Role of gammadelta $\mathrm{T}$ lymphocytes in the development of Behçet's disease. Clin Exp Immunol 107: 241-247, 1997. 